\title{
Microextração em fase sólida de 6-pentil- $\alpha$-pirona produzida por fermentação em estado sólido
}

\author{
Solid-phase microextraction of 6-pentyl-a-pyrone produced by solid-state fermentation
}

\author{
Aline de Souza RAMOS ${ }^{1,2 *}$, Paula Salles de Oliveira MARTINS ${ }^{1,3}$, \\ Sorele Batista FIAUX ${ }^{4}$, Selma Gomes Ferreira LEITE ${ }^{1}$
}

\begin{abstract}
Resumo
A substância 6-pentil- $\alpha$-pirona (6-PP) é uma lactona com aroma característico de coco e uso aprovado pela agência americana Food and Drug Administration (FDA). O presente trabalho teve como objetivo ajustar as condições de microextração em fase sólida em headspace para a quantificação por cromatografia gasosa de 6-PP produzida pelo fungo Trichoderma harzianum a partir do pó da casca de coco verde. Para isto, foi realizado um estudo em que soluções padronizadas de 6-PP foram adicionadas ao pó da casca de coco verde. A substância foi extraída por uma fibra de polidimetilsiloxano. Agitação e adição de solução de $\mathrm{NaCl}$ a $25 \%$ (p/v) resultaram em melhor reprodutibilidade nas análises. Um planejamento composto central foi empregado para estudar três fatores: temperatura de extração, tempo de condicionamento e tempo de extração. Boas respostas foram obtidas com a temperatura de $79^{\circ} \mathrm{C}$ e tempo de extração de 29 minutos. O efeito da variável tempo de condicionamento não foi significativo e foi fixado em 2 minutos. Após o desenvolvimento do método analítico, este foi empregado para determinar a produção de 6-PP por fermentação em estado sólido realizada por Trichoderma harzianum e com o uso de pó da casca de coco verde como suporte para o processo.

Palavras-chave: aroma de coco; 6-pentil- $\alpha$-pirona; fermentação em estado sólido; MEFS; pó da casca de coco verde; planejamento experimental.
\end{abstract}

\begin{abstract}
The lactone 6-pentyl- $\alpha$-pyrone (6-PP) is a coconut-like aroma compound approved by the American agency Food and Drug Administration (FDA) for food use. The aim of the present study was to improve the extraction conditions of headspace solid-phase microextraction coupled with gas chromatography to quantify the 6-PP produced by Trichoderma harzianum using green coir dust. To achieve this purpose, standard solutions of 6-PP were added to green coir dust. The compound was extracted by a polydimethylsiloxane fiber. Agitation and addition of a $25 \%$ $\mathrm{NaCl}$ solution (w/v) resulted in better reproducibility. A central composite design was applied to study three factors: extraction temperature, conditioning time, and extraction time. Good responses were obtained when the extraction temperature was $79^{\circ} \mathrm{C}$, and the extraction time was 29 minutes. The conditioning time effect was not significant and was established at 2 minutes. After the development of the analytical method, it was employed to determine the 6-PP production by Trichoderma harzianum in solid-state fermentation using green coir dust as the support material for the process.

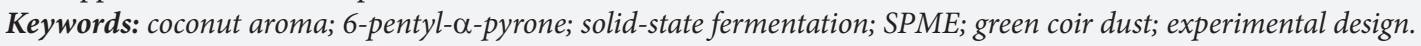

\section{Introdução}

Compostos aromatizantes são extremamente importantes para a indústria alimentícia e farmacêutica, pois aumentam a aceitação dos produtos pelos consumidores. A substância 6-pentil- $\alpha$-pirona (6-PP) é uma lactona com aroma característico de coco e que tem seu uso em alimentos aprovado pela agência americana Food and Drug Administration (FDA) (BONNARME et al., 1997; SERRANO-CARREÓN et al., 2004). Alguns fungos do gênero Trichoderma são capazes de produzir a substância que também apresenta potente atividade antifúngica e tem baixa toxicidade a mamíferos (COONEY et al., 1997). Sua síntese química é difícil: requer sete etapas reacionais e alta temperatura. Um processo fermentativo oferece a possibilidade de condições brandas de reação e permite o uso do termo de conotação positiva "aroma natural" (SARHYBAGNON et al., 2000). Entretanto, as concentrações obtidas com o uso de microrganismos são geralmente baixas, provavelmente devido ao efeito inibitório no metabolismo fúngico (PRAPULLA et al., 1992).

Concentrações mais elevadas de aromas podem ser conseguidas quando a fermentação é conduzida em meio sólido (FÉRON; BONNARME; DURAND, 1996). A fermentação em estado sólido permitiu que Sarhy-Bagnon et al. (2000) e Araujo, Pastore e Berger (2002) obtivessem cerca de $3 \mathrm{mg}$ de

\footnotetext{
Recebido para publicação em 27/10/2007

Aceito para publicação em 7/4/2008 (002964)

Departamento de Engenharia Bioquímica, Ilha do Fundão, Centro de Tecnologia, Escola de Química, Universidade Federal do Rio de Janeiro - UFRJ, CEP 21949-900,

Rio de Janeiro - RJ, Brasil. E-mail: ramos.aline@gmail.com

${ }^{2}$ Fundação Oswaldo Cruz - FIOCRUZ, Farmanguinhos, Rua Sizenando Nabuco, 100, CEP 21041-250, Manguinhos, Rio de Janeiro - RJ, Brasil

3 Instituto Nacional da Propriedade Industrial - INPI, Rua Mayrink Veiga, 9, CEP 20090-910, Centro, Rio de Janeiro - RJ, Brasil

${ }^{4}$ Faculdade de Farmácia, Universidade Federal Fluminense - UFF, Rua Mário Viana, 523, CEP 24241-000, Santa Rosa, Niterói - RJ, Brasil

${ }^{*}$ A quem a correspondência deve ser enviada
} 
6-PP/ g de matéria seca (MS) usando bagaço de cana-de-açúcar impregnado com uma solução nutriente. Essa é a produção máxima descrita na literatura até o momento.

A extração de 6-PP para posterior quantificação por cromatografia gasosa tem sido realizada por codestilação com diclorometano (BONNARME et al., 1997; SERRANOCARREÓN et al., 1992) ou por extração por solvente de baixa polaridade, como diclorometano (KALYANI; PRAPULLA; KARANTH, 2000), éter (PRAPULLA et al., 1992) e hexano (SARHY-BAGNON et al., 2000). Esses métodos são baseados em tecnologias tradicionais laboriosas e que exigem o uso de solventes tóxicos. Uma alternativa é a microextração em fase sólida (MEFS), que necessita de menor número de etapas e tempo reduzido para a preparação da amostra. Também tem a grande vantagem de não utilizar solventes, sendo considerada uma tecnologia mais limpa (KATAOKA; LORD; PAWLISZYN, 2000). Como tem alta sensibilidade, a MEFS pode ser aplicada na detecção de aromas e é capaz de transpor as dificuldades relacionadas com a baixa concentração e perdas por volatilização (DÍAZ et al., 2002; PELLATI et al., 2005).

$\mathrm{Na}$ MEFS, um suporte sólido contendo uma pequena quantidade da fase extratora é mantido em contato com uma amostra por um período de tempo suficiente para que seja atingido um equilíbrio de concentrações entre as fases. Em seguida deve ser feita a dessorção, em geral realizada termicamente no injetor de um cromatógrafo a gás (CG), e subsequente análise (LORD; PAWLISZYN, 2000). Nas amostras sólidas, a MEFS deve ser realizada em headspace. $\mathrm{O}$ transporte dos analitos para a fase gasosa pode ser melhorado pelo aquecimento da amostra e/ou adição de solução aquosa contendo um sal solúvel, como cloreto de sódio. As condições mais adequadas de extração devem ser determinadas experimentalmente (PROSEN; ZUPANČIČ-KRALJ, 1999).

O objetivo deste trabalho foi estabelecer as condições de MEFS em headspace para a quantificação por cromatografia gasosa de 6-PP adicionado ao pó da casca de coco verde. Após o desenvolvimento do método analítico, este foi aplicado para avaliar o potencial de produção de 6-PP por duas linhagens de Trichoderma harzianum em fermentação em estado sólido usando o pó da casca de coco verde como suporte para o processo.

\section{Material e métodos}

\subsection{Material}

O pó da casca de coco verde, cedido pela Embrapa Agroindústria Tropical (Fortaleza, CE, Brasil), foi obtido por trituração da casca de coco verde (Cocus nucifera), secagem ao sol e classificação. O pó com granulometria inferior a 1,19 mm (14 mesh Tyler) foi empregado como suporte para a fermentação. Ágar-batata-dextrose foi obtido de Himedia Laboratories (Mumbai, Índia). A lactona 6-PP, com 96\% de pureza, foi adquirida de Sigma-Aldrich (St. Louis, MO, USA). Todo o material empregado para MEFS (fibras revestidas por polidimetilsiloxano com $100 \mu \mathrm{m}$ de espessura, aparato para injeção manual em CG, recipientes de vidro com capacidade para $40 \mathrm{~mL}$, tampas de polipropileno e septos de teflon/silicone) foi adquirido de Supelco (Bellefonte, PA, USA).

\subsection{Microrganismo e condições de cultura}

As linhagens de T. harzianum (4040 e 4042) foram obtidas da coleção de culturas do Departamento de Micologia, Instituto Oswaldo Cruz (IOC/FIOCRUZ). Para a produção de esporos, os fungos foram cultivados em meio ágar-batata-dextrose durante sete dias a $28^{\circ} \mathrm{C}$. Os esporos obtidos foram ressuspensos em solução salina $(0,9 \% \mathrm{NaCl})$ e quantificados em câmara de Neubauer. Frascos Erlenmeyer $(250 \mathrm{~mL})$ contendo 4,5 g de pó da casca de coco verde umedecido com $4,5 \mathrm{~mL}$ de solução nutriente foram inoculados com $1 \mathrm{~mL}$ de uma suspensão de esporos contendo $10^{6}$ esporos/mL e incubados a $28^{\circ} \mathrm{C}$, sem agitação. A solução nutriente tinha a seguinte composição (g. $\left.\mathrm{L}^{-1}\right)$ : glicose, 30; $\left(\mathrm{NH}_{4}\right)_{2} \mathrm{SO}_{4}, 0,943$; extrato de levedura 1,0; $\mathrm{MgSO}_{4} .7 \mathrm{H}_{2} \mathrm{O}, 5 ; \mathrm{KH}_{2} \mathrm{PO}_{4}, 1,0 ; \mathrm{KCl}, 0,5 ; \mathrm{CaCl}_{2} .2 \mathrm{H}_{2} \mathrm{O}, 0,008$; $\mathrm{FeSO}_{4} .7 \mathrm{H}_{2} \mathrm{O}, 0,01 ; \mathrm{ZnSO}_{4} .7 \mathrm{H}_{2} \mathrm{O}, 0,001$. Suporte e solução nutriente foram autoclavados separadamente, a $115^{\circ} \mathrm{C}$ durante 20 minutos. Todos os cultivos foram realizados em triplicata, sendo um frasco por amostra.

\subsection{Métodos analíticos}

Para a extração de 6-PP das amostras, empregou-se a técnica de microextração em fase sólida em headspace, com fibras revestidas por polidimetilsiloxano (PDMS) com $100 \mu \mathrm{m}$ de espessura. As fibras foram preparadas para o uso segundo as instruções do fabricante. A amostra (0,250 g) adicionada de $20 \mathrm{~mL}$ de solução de $\mathrm{NaCl}$ a $25 \%(\mathrm{p} / \mathrm{v})$ foi acondicionada em recipiente com $40 \mathrm{~mL}$ de capacidade hermeticamente fechado com tampa de polipropileno e septo de teflon/silicone. O conjunto foi imerso em banho controlado para manutenção da temperatura durante o condicionamento e a extração. A amostra foi mantida em suspensão por agitação magnética constante. Após um período de tempo (tempo de condicionamento), o aparato de MEFS foi manualmente introduzido no recipiente contendo a amostra e a fibra foi exposta durante o tempo de extração. Tempo de condicionamento, tempo de extração e temperatura foram estudados conforme o planejamento experimental descrito no item a seguir.

Imediatamente após a extração, os analitos sofreram dessorção térmica a $250{ }^{\circ} \mathrm{C}$ durante 4 minutos no injetor do cromatógrafo a gás (Varian Inc., modelo CP3800, Palo Alto, CA, USA), onde foram analisados. Ensaios em branco (pó da casca de coco verde sem a adição de 6-PP) realizados após as análises de amostras contendo até $1,0 \mathrm{mg} \cdot \mathrm{g}^{-1} \mathrm{MS}$ indicaram que a exposição da fibra a $250^{\circ} \mathrm{C}$ durante 4 minutos foi suficiente para a dessorção completa dos analitos, não tendo sido observada contaminação cruzada. Os analitos foram separados em coluna CP Sil 5CB (30 m de comprimento, 0,32 mm de diâmetro interno e $0,25 \mu \mathrm{m}$ de espessura de fase). O modo splitless foi aplicado nos primeiros 4 minutos para a dessorção e durante o restante do tempo de corrida empregou-se taxa de split de 1/35. A temperatura da coluna foi mantida a $35{ }^{\circ} \mathrm{C}$ nos primeiros 4 minutos e em seguida elevada a uma taxa de $10^{\circ} \mathrm{C} / \mathrm{min}$ até $130{ }^{\circ} \mathrm{C}$, tendo sido mantida constante por 2 minutos e então aquecida até $240{ }^{\circ} \mathrm{C}$ a uma taxa de $40{ }^{\circ} \mathrm{C} / \mathrm{min}$. A detecção foi 
realizada por ionização de chama a $250{ }^{\circ} \mathrm{C}$. A vazão do gás de arraste $\left(\mathrm{N}_{2}\right)$ foi de $1,2 \mathrm{~mL} / \mathrm{min}$. $\mathrm{O}$ composto foi identificado por comparação com o tempo de retenção do padrão de 6-PP sob as mesmas condições de análise.

A quantificação foi feita por padronização externa. Uma curva-padrão foi construída com sete níveis de análises em triplicata de pó da casca de coco verde $(0,250 \mathrm{~g})$ impregnado com soluções-padrão de 6-PP. As soluções-padrão foram preparadas por diluição de 6-PP (Sigma-Aldrich, St. Louis, MO, USA) com etanol-água destilada $(1: 100 \mathrm{v} / \mathrm{v})$. O intervalo de concentrações foi de 0,04 a 1,2 mg de 6-PP/g MS. O gráfico foi construído com o emprego do software Microsoft Office ${ }^{\varpi x c e l}$ 2003. A curva foi linear $\left(\mathrm{r}^{2}=0,99\right)$, passou pela origem e cobriu a faixa de concentração das amostras. A MEFS para a construção da curva-padrão foi realizada segundo as melhores condições determinadas pelo planejamento experimental.

\subsection{Planejamento experimental para padronização das condições de MEFS para quantificação de 6-PP}

Através de um planejamento composto central, foram estudadas as variáveis: tempo de condicionamento ( $t c, \mathrm{~min})$, tempo de extração (text, min) e temperatura de extração $\left(T,{ }^{\circ} \mathrm{C}\right)$. O planejamento consistiu de 20 experimentos distribuídos aleatoriamente: um fatorial $2^{3}, 6$ pontos axiais e 6 replicatas do ponto central. Os níveis estudados e o domínio experimental estão apresentados na Tabela 1. Análises foram efetuadas com amostras-padrão, compostas de 0,250 g de pó da casca de coco verde impregnado com $0,500 \mathrm{~mL}$ de uma solução contendo 30 mg. $L^{-1}$ de 6-PP. Esta solução foi preparada por diluição de 6-PP (Sigma-Aldrich, St. Louis, MO, USA) em etanol-água destilada $(1: 100 \mathrm{v} / \mathrm{v})$. A variável de resposta foi a área do pico correspondente à análise cromatográfica de 6-PP. A análise estatística foi realizada com o emprego do software Statistica 6.0 (StatSoft Inc., Tulsa, OK, USA). Um experimento em triplicata foi conduzido nas melhores condições indicadas pelo planejamento para confirmar a previsão do modelo.

\section{Resultados e discussão}

\subsection{Padronização das condições de MEFS para a quantificação de 6-PP}

As análises de compostos de aroma costumam ser difíceis principalmente pela alta volatilidade e baixas concentrações nas amostras. Bons resultados têm sido conseguidos com o emprego da técnica de MEFS usando fibras revestidas com PDMS de

Tabela 1. Fatores e domínios experimentais estudados no planejamento composto central empregado no ajuste das condições de MEFS em análises de 6-PP no pó da casca de coco verde.

\begin{tabular}{|c|c|c|c|c|c|}
\hline \multirow[t]{2}{*}{ Fatores } & \multicolumn{5}{|c|}{ Níveis $^{\mathrm{a}}$} \\
\hline & $-\alpha$ & - & 0 & + & $+\alpha$ \\
\hline Tempo de condicionamento $\left(t_{c}, \min \right)$ & 0 & 4 & 10 & 16 & 20 \\
\hline Tempo de extração $\left(\mathrm{t}_{\mathrm{ex}}, \mathrm{min}\right)$ & 3 & 10 & 20 & 30 & 37 \\
\hline Temperatura $\left(\mathrm{T},{ }^{\circ} \mathrm{C}\right)$ & 35 & 45 & 60 & 75 & 85 \\
\hline
\end{tabular}

$100 \mu \mathrm{m}$ de espessura, um material apolar (KATAOKA; LORD; PAWLISZYN, 2000). Segundo Alpendurada (2000), PDMS é o revestimento mais popular e deve ser empregado sempre que possível, pois é capaz de sofrer dessorção a temperaturas de até $300{ }^{\circ} \mathrm{C}$ e também pode ser empregado para extrair algumas substâncias polares, desde que as condições de extração sejam otimizadas.

Para aplicar a MEFS nas análises de 6-PP foi necessário determinar as melhores condições de extração. Para aumentar a reprodutibilidade, foram adicionados $20 \mathrm{~mL}$ de uma solução a $25 \%$ de $\mathrm{NaCl}$ à amostra e a mistura foi submetida a agitação constante através de uma barra magnética. A agitação permite que o equilíbrio vapor-líquido seja atingido mais rapidamente e a adição de sais solúveis à amostra diminui a solubilidade de compostos orgânicos em meio aquoso devido ao efeito salting out (PROSEN; ZUPANČIČ-KRALJ, 1999).

Em seguida, temperatura de extração, tempo de condicionamento e tempo de extração foram estudados. A temperatura de extração tem duplo impacto nas análises: temperaturas elevadas permitem a rápida liberação dos analitos para a fase gasosa, mas os coeficientes de partição são reduzidos (PROSEN; ZUPANČIČ-KRALJ, 1999). Como a transferência de calor no headspace é menor do que na amostra, o aquecimento da fibra deve ser mais lento. O ideal é que o tempo de extração seja suficiente para o equilíbrio entre as fases ser alcançado, porém sem aquecer demasiadamente a fibra (ULRICH, 2000). Para aumentar a concentração dos analitos no headspace e evitar dessorção indesejada, a amostra também pode ser aquecida antes da exposição da fibra (tempo de condicionamento). Sendo assim, temperatura de extração, tempo de condicionamento e tempo de extração estão relacionados e são frequentemente investigados em estudos de otimização (DÍAZ et al., 2002; PELLATI et al., 2005; PRADO; GARRIDO; PERIAGO, 2004).

Para o estudo das três variáveis, foi aplicado um planejamento composto central. Este tipo de planejamento é adequado para avaliar o efeito de algumas variáveis simultaneamente e permite a introdução de termos quadráticos ao modelo, fundamentais para a descrição de superfícies de resposta curvas. Por gerar um grande número de experimentos, é recomendável apenas para estudar poucos fatores (ZEAITER et al., 2004). Já foi empregado por Díaz et al. (2002) e Pellati et al. (2005) para otimizar as condições de MEFS em headspace. O domínio experimental foi escolhido com base nos estudos descritos na literatura para análises de compostos voláteis (DÍAZ et al., 2002; KATAOKA; LORD; PAWLISZYN, 2000; LIU; YANG, 2002; MILLS; WALKER, 2000; PELLATI et al., 2005). Além disso, ensaios preliminares demonstraram que temperaturas de extração inferiores a $30{ }^{\circ} \mathrm{C}$ e superiores a $90{ }^{\circ} \mathrm{C}$ conduziram a valores extremamente baixos para a variável de resposta (dados não apresentados). Os resultados para os 20 experimentos estão apresentados na Tabela 2.

A análise de variância (ANOVA) dos resultados permitiu verificar a significância estatística de cada variável e das interações entre as variáveis. O nível de significância das análises foi estabelecido em 95\%. A Figura 1 apresenta o diagrama de Pareto para a variável de resposta. 
Apenas os termos lineares e quadráticos relacionados com a temperatura e o tempo de extração foram significativos no domínio estudado $(\mathrm{p}<0,05)$. O desvio padrão relativo, calculado a partir das replicatas do ponto central, foi 9,6\%. A Equação 1 descreve a área do pico cromatográfico correspondente ao 6-PP em função das variáveis significativas normalizadas.

Tabela 2. Condições experimentais e variável de resposta do planejamento composto central empregado na escolha das condições de MEFS em análises de 6-PP no pó da casca de coco verde.

\begin{tabular}{ccccc}
\hline Exp. & $\mathrm{t}_{\mathrm{c}}(\min )$ & $\mathrm{t}_{\mathrm{ex}}(\min )$ & $\mathrm{T}\left({ }^{\circ} \mathrm{C}\right)$ & Área $(\mathrm{mV} . \mathrm{s})$ \\
\hline 1 & 4 & 10 & 45 & 15,759 \\
2 & 4 & 10 & 75 & 59,676 \\
3 & 4 & 30 & 45 & 40,981 \\
4 & 4 & 30 & 75 & 91,109 \\
5 & 16 & 10 & 45 & 15,558 \\
6 & 16 & 10 & 75 & 67,255 \\
7 & 16 & 30 & 45 & 34,412 \\
8 & 16 & 30 & 75 & 82,425 \\
9 & 0 & 20 & 60 & 55,179 \\
10 & 20 & 20 & 60 & 63,944 \\
11 & 10 & 3 & 60 & 17,904 \\
12 & 10 & 37 & 60 & 71,887 \\
13 & 10 & 20 & 35 & 9,741 \\
14 & 10 & 20 & 85 & 71,329 \\
15 & 10 & 20 & 60 & 65,035 \\
16 & 10 & 20 & 60 & 60,904 \\
17 & 10 & 20 & 60 & 72,511 \\
18 & 10 & 20 & 60 & 67,229 \\
19 & 10 & 20 & 60 & 78,528 \\
20 & 10 & 20 & 60 & 63,215 \\
\hline
\end{tabular}

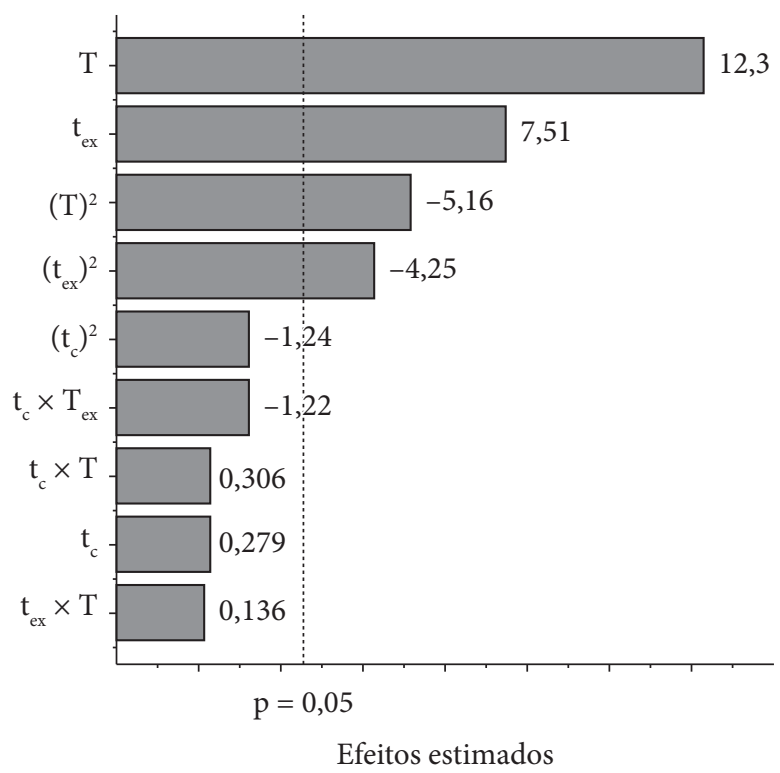

Figura 1. Diagrama de Pareto para a variável de resposta (área do pico cromatográfico correspondente a 6-PP). Legenda: $t$ : tempo de condicionamento, $\mathrm{t}_{\mathrm{ex}}$ : tempo de extração, $\mathrm{T}$ : temperatura.
Área $=66+13 \times t_{e x}-7,0 \times t_{e x}^{2}+22 \times T-8,8 \times T^{2}$

O elevado coeficiente de determinação $\left(\mathrm{r}^{2}=0,94\right.$ e $\mathrm{r}^{2}$ ajustado $=0,92$ ), o alto valor de $\mathrm{p}$ associado com a falta de ajuste do modelo $(\mathrm{p}=0,5)$ e a distribuição normal dos resíduos segundo os testes de Kolmogorov-Sminorv e Shapiro-Wilk indicam bom ajuste do modelo aos dados experimentais.

A Figura 2 mostra os efeitos da temperatura e do tempo de extração na área do pico cromatográfico correspondente a 6-PP. Os termos relacionados à variável tempo de condicionamento não foram significativos $(\mathrm{p}>0,05)$ e não foram incluídos no modelo. Este resultado indica que, dentro do domínio experimental investigado, o estado de equilíbrio entre as fases pode ser atingido independentemente do pré-aquecimento da amostra no recipiente hermeticamente fechado antes da exposição da fibra. As melhores condições de extração (pontos de máximo do modelo) foram tempo de extração de 29 minutos e temperatura de $79^{\circ} \mathrm{C}$, determinados através da resolução de um sistema de equações formado pelas derivadas parciais da Equação 1 igualadas a zero. O tempo de condicionamento foi estabelecido em 2 minutos.

Uma análise em triplicata foi realizada para verificar se o modelo poderia prever a área do pico cromatográfico correspondente a 6-PP nas melhores condições de extração indicadas. As áreas dos picos (mV.s) foram 79, 82 e 69. Apesar de um resultado melhor $(91 \mathrm{mV} . \mathrm{s})$ ter sido encontrado no planejamento em condições diferentes (ver Tabela 2), esses valores estavam dentro do intervalo de $95 \%$ de previsão. Como as áreas dos picos cromatográficos foram consideradas satisfatórias e o desvio padrão relativo foi de $9 \%$, as condições de extração indicadas pelo modelo foram empregadas em análises

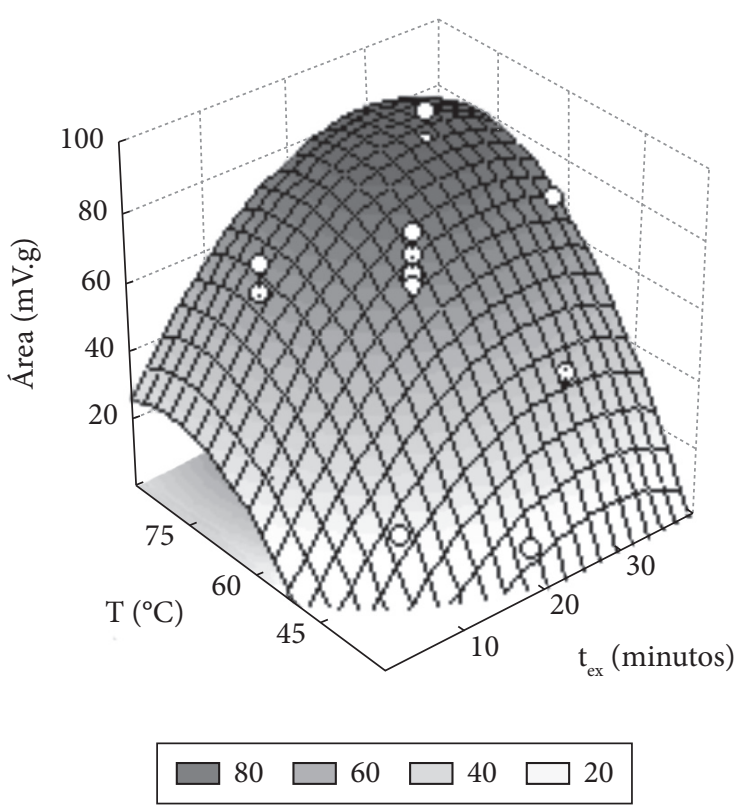

Figura 2. Área do pico correspondente a 6-PP em função da temperatura (T) e do tempo de extração $\left(t_{e x}\right)$. Tempo de condicionamento: 2 minutos. 


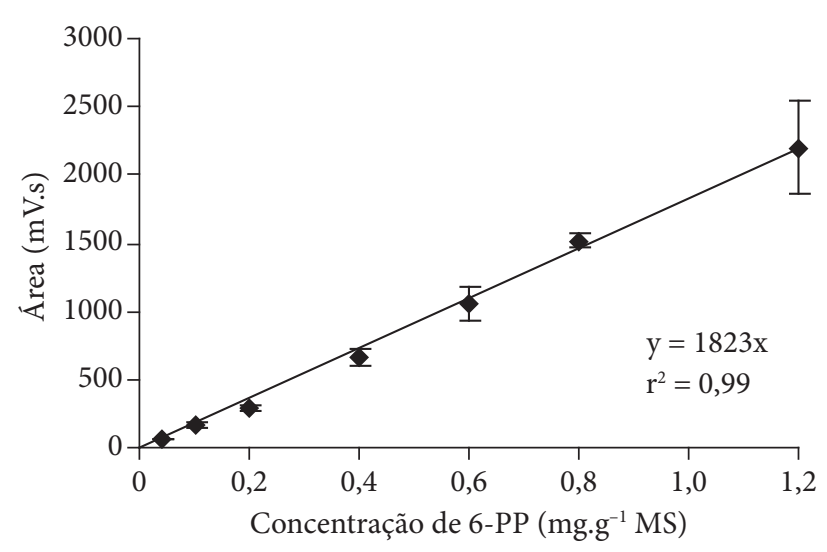

Figura 3. Curva-padrão para quantificação de 6-PP presente no pó da casca de coco verde por MEFS em headspace.

posteriores. A curva-padrão (Figura 3) variando de 0-1,2 mg de 6-PP/g MS, posteriormente construída, apresentou ajuste linear $\left(r^{2}=0,99\right)$ na faixa de concentrações estudada. Portanto, a técnica de MEFS se mostrou adequada para a análise de 6-PP presente no pó da casca de coco verde.

\subsection{Aplicação do método para a análise da produção de 6-PP por fermentação em estado sólido}

Neste estudo, duas linhagens de T. harzianum (4040 e 4042) foram comparadas quanto à produção de 6-PP. Experimentos em triplicata foram realizados nas mesmas condições para as duas linhagens. Após cinco dias de cultivo, T. harzianum 4040 produziu $0,5 \pm 0,1 \mathrm{mg}$ de $6-\mathrm{PP} / \mathrm{g} \mathrm{MS}$, enquanto foi obtido 0,3 $\pm 0,1 \mathrm{mg}$ de 6-PP/g MS com o emprego de T. harzianum 4042. A diferença foi apenas marginalmente significativa $(p=0,07)$. Esses resultados indicam que as duas linhagens têm equivalente potencial para a produção de 6-PP em meio sólido.

Em comparação com T. harzianum 4040, a linhagem 4042 apresentou crescimento mais rápido e maior formação de esporos quando cultivada em meio ágar-batata-dextrose. A linhagem 4042 foi então avaliada quanto à produção de 6-PP também após 3, 7, 9 e 11 dias de cultivo. Após três dias de cultivo, a presença de 6-PP foi detectada, porém em baixa concentração $\left(0,01 \pm 0,003 \mathrm{mg} \cdot \mathrm{g}^{-1} \mathrm{MS}\right)$. A concentração máxima foi conseguida após 7 dias de cultivo $\left(1,0 \pm 0,05 \mathrm{mg}^{-1} \mathrm{~g}^{-1} \mathrm{MS}\right)$ e se manteve constante durante o restante do tempo estudado.

\section{Conclusões}

A metodologia de MEFS em headspace desenvolvida neste trabalho, associada à análise por cromatografia gasosa, permitiu analisar o composto 6-PP presente em amostras de pó de casca de coco verde. As condições padronizadas também foram empregadas com êxito na determinação da produção deste composto por duas linhagens de T. harzianum em fermentação em estado sólido, o que permitiu verificar o potencial produtivo de ambas as linhagens. A técnica se mostrou promissora como método analítico a ser empregado no desenvolvimento do processo de produção de 6-PP, composto de alto valor agregado, principalmente se for obtido por fermentação, por permitir o uso do termo "aroma natural".

\section{Agradecimentos}

O presente trabalho foi realizado com o apoio do Conselho Nacional de Desenvolvimento Científico e Tecnológico (CNPqBrasil), da Fundação Universitária José Bonifácio (FUJB) e da Fundação Carlos Chagas Filho de Amparo à Pesquisa do Estado do Rio de Janeiro (FAPERJ).

\section{Referências bibliográficas}

ALPENDURADA, M. F. Solid-phase microextraction: a promising technique for sample preparation in environmental analysis. Journal of Chromatography A, v. 889, n. 1-2, p. 3-14, 2000.

ARAUJO, A. A.; PASTORE, G. M.; BERGER, R. G. Production of coconut aroma by fungi cultivation in solid-state fermentation. Applied Biochemistry and Biotechnology, v. 98-100, n. 1-9, p. 747-751, 2002.

BONNARME, P. et al. Production of 6-pentyl- $\alpha$-pyrone by Trichoderma sp. from vegetable oils. Journal of Biotechnology, v. 56, n. 2, p. 143-150, 1997.

COONEY, J. M. et al. Effect of solid substrate, liquid supplement, and harvest time on 6-n-pentyl-2H-pyran-2-one (6PAP) production by Trichoderma spp. Journal of Agricultural and Food Chemistry, v. 45, n. 2, p. 531-534, 1997.

DÍAZ, P. et al. Truffle aroma analysis by headspace solid phase microextraction. Journal of Agricultural and Food Chemistry, v. 50, n. 22, p. 6468-6472, 2002.

FÉRON, G.; BONNARME, P.; DURAND, A. Prospects for the microbial production of food flavours. Trends in Food Science \& Technology, v. 7, n. 9, p. 285-293, 1996.

KALYANI, A.; PRAPUlla, S. G.; KARANTH, N. G. Study on the production of 6-pentyl- $\alpha$-pyrone using two methods of fermentation. Applied Microbiology and Biotechnology, v. 53, n. 5, p. 610-612, 2000.

KATAOKA, H.; LORD, H. L.; PAWLISZYN, J. Applications of solidphase microextraction in food analysis. Journal of Chromatography A, v. 880, n. 1-2, p. 35-62, 2000.

LIU, T. T.; YANG, T. S. Optimization of solid-phase microextraction analysis for studying change of headspace flavor compounds of banana during ripening. Journal of Agricultural and Food Chemistry, v. 50, n. 4, p. 653-657, 2002.

LORD, H.; PAWLISZYN, J. Evolution of solid-phase microextraction technology. Journal of Chromatography A, v. 885, n. 1-2, p. 153-193, 2000.

MILLS, G. A.; WALKER, V. Headspace solid-phase microextraction procedures for gas chromatographic analysis of biological fluids and materials. Journal of Chromatography A, v. 902, n. 1, p. 267-287, 2000.

PELLATI, F. et al. Headspace solid-phase microextraction-gas chromatography-mass spectrometry analysis of the volatile compounds of Evodia species fruits. Journal of Chromatography A, v. 1087, n. 1-2, p. 265-273, 2005.

PRADO, C.; GARRIDO, J.; PERIAGO, J. F. Urinary benzene determination by SPME/GC-MS: A study of variables by fractional factorial design and response surface methodology. Journal of Chromatography B, v. 804, n. 2, p. 255-261, 2004. 
PRAPULLA, S. G. et al. Production of 6-pentyl-a-pyrone by Trichoderma viride. Flavour and Fragrance Journal, v. 7, n. 4, p. 231-234, 1992.

PROSEN, H.; ZUPANČIČ-KRALJ, L. Solid-phase microextraction. Trends in Analytical Chemistry, v. 18, n. 4, p. 272-282, 1999.

SARHY-BAGNON, V. et al. Production of 6-pentyl- $\alpha$-pyrone by Trichoderma harzianum in liquid and solid state cultures. Process Biochemistry, v. 36, n. 1-2, p. 103-109, 2000.

SERRANO-CARREÓN, L. et al. Rhizoctonia solani, an elicitor of 6 -pentyl- $\alpha$-pyrone production by Trichoderma harzianum in a two liquid phases, extractive fermentation system. Biotechnology Letters, v. 26, n. 18, p. 1403-1406, 2004.

SERRANO-CARREÓN, L. et al. Production of 6-pentyl- $\alpha$-pyrone by Trichoderma harzianum from 18: n fatty acid methyl esters. Biotechnology Letters, v. 14, n. 11, p. 1019-1024, 1992.

ULRICH, S. Solid-phase microextraction in biomedical analysis. Journal of Chromatography A, v. 902, n. 1, p. 167-194, 2000.

ZEAITER, M. et al. Robustness of models developed by multivariate calibration. Part I: The assessment of robustness. Trends in Analytical Chemistry, v. 23, n. 2, p. 157-170, 2004. 